\title{
Linear and Non-Linear Synthesis of Unequally Spaced Time-Modulated Linear Arrays Using Evolutionary Algorithms
}

\author{
Somnath PATRA ${ }^{1,2}$, Sujit Kumar MANDAL ${ }^{2}$, Gautam Kumar MAHANTI ${ }^{2}$, Narendra Nath PATHAK ${ }^{3}$ \\ ${ }^{1}$ Dept. of Electronics and Communication Engineering, SMIT (TIG), Guptipara, Hooghly, WB, India \\ ${ }^{2}$ Dept. of Electronics and Communication Engineering, NIT Durgapur, WB, India \\ ${ }^{3}$ Dept. of Electronics and Communication Engineering, Dr. B. C. Roy Engineering College, Durgapur, WB, India
}

somnath.tech@gmail.com,skmandal2006@gmail.com,gkm@ece.nitdgp.ac.in,narendra.pathak@bcrec.ac.in

Submitted February 7, 2021 / Accepted May 15, 2021

\begin{abstract}
A novel method of designing unequally spaced time-modulated arrays (UESTMAs) by handling fewer optimization parameters with reduced problem dimension is presented in this paper. For synthesizing UESTMA, two design parameters, specifically, the non-linear parameterelement position, and the linear parameter - on-time durations are optimized in two steps. Different possible cases of linear and non-linear synthesis methods such as, positiononly (PO), on-time only (OTO), position then on-time (PTOT), on-time then position (OTTP), and simultaneous position on-time (SPOT) are considered. To examine the performance of the synthesis methods, three global search stochastic algorithms based on differential evolution (DE), teaching-learning-based optimization (TLBO) and quantum particle swarm optimization (QPSO) have been employed to achieve the array pattern with significantly suppressed side lobe levels and sideband levels. Through comparative study, it is observed that the two step nonliner to linear synthesis method by fewer optimization parameters is efficient to provide better pattern with less computation time.
\end{abstract}

\section{Keywords}

Time-modulation, position-only, on-time only, position on-time, side lobe level, sideband level

\section{Introduction}

Suppression of side lobe level (SLL) plays a vital role for designing antenna arrays and it has been extensively studied over several years. Theoretically, SLL of the array pattern can be suppressed to a desired value by providing proper excitation amplitude distribution in the array elements. There are various numerical and analytical techniques such as Dolph-Chebyshev and Taylor series methods [1] which are used to synthesize low side lobe antenna array patterns over the past years. These numerical meth- ods are applicable for equally spaced antenna arrays and are employed to realize non-uniform excitation amplitude distribution of the desired pattern. However, high dynamic range ratio (DRR) of non-uniform amplitude excitation as required to reduce SLL in equally spaced antenna arrays increases the complexity and cost of the feed network [2], [3]. In contrast to equally spaced antenna arrays, in the recent past years, unequally spaced antenna array has been found to be effective to provide low side lobe patterns with uniform amplitude excitation [4]. The additional advantage of using unequally spaced antenna array is that the low side lobe pattern can be obtained with a smaller number of antenna elements for a given aperture size [5].

However, in 1959, H. E. Shanks [6] first proposed time-modulation to synthesize power pattern in antenna arrays by periodically controlling ON-OFF switching sequence of the radiating elements by using high-speed RF switches. The periodical switching of the antenna elements with some predetermined timing sequence leads to introduce 'time' as a 'fourth dimension' for synthesizing antenna array patterns with low and ultra-low values of SLL [7], [8]. The simple ON-OFF switching mechanism with optimized time pulse enables to suppress the SLL of the power pattern even with uniform excitation amplitude [9] and is effective in synthesizing different power patterns such as, sum and difference pattern [10], and flat-top shaped beam pattern [11]. It is to be noted that, in traditional amplitude tapering method, due to the various systematic errors, the array elements are not possible to feed with the exact value of the excitation amplitude of the required pattern. As a result, practically, it is exceedingly difficult to realize low/ultra-low sidelobe pattern with high DRR of static excitation amplitude in conventional antenna arrays (CAAs). On the other hand, power pattern in timemodulated arrays (TMAs) is controlled by using a set of switch-on time sequence of the array elements. Accordingly, the exact value of the on-time sequence for the low sidelobe patterns in TMA can be accurately maintained with the help of software. However, the sideband radiation due to the periodical commutation of the antenna elements 
is the major problem in TMAs. Such sideband radiated signal may be appeared as one unwanted interfering signal to the other communication channel. Also, the power wastage in the form of sideband radiation leads to reduce the overall directivity of the antenna array. In this regard, many nature inspired global optimization algorithms like, differential evolution (DE) [12-14], artificial bee colony (ABC) [4], simulated annealing (SA) [8], genetic algorithm (GA) [15], particle swarm optimization (PSO) [16], and recently, a hybrid multi-objective optimization using PSO and convex programming [17] are used to obtain desired power patterns by simultaneously minimizing the SLL and the relative sideband maximum with respect to the center frequency pattern maximum, termed as, sideband level (SBL) to sufficiently low values. Also, in some application it is shown that the sidebands of TMAs are useful to generate simultaneous multiple beam patterns [16], [18]. All the evolutionary and swarm intelligent based algorithms are generally dealt with stochastically generated population with some common parameters such as population size and number of generations. The performance of such population based heuristic algorithms depends on few algorithm specific controlling parameters. For example, the parameters of DE are crossover probability, mutation intensity and selection; the PSO parameters are social constant, cognitive coefficient and inertia weight; and for $\mathrm{ABC}$, these are number of onlooker bees, employed bees, scout bees and limit. The controlling parameters of the algorithm serve an important role and unambiguously effect on the performance, as improper tuning of the controlling parameters leads to increase the computational burden as well as provide undesired results. Considering this fact, Rao et al. (2011) introduced a population-based algorithm known as teaching-learning-based optimization (TLBO) [19], [20] that does not require any such tuning parameters while only the common parameters are sufficient for its functioning.

However, synthesis of UESTMA is a non-linear, multimodal problem and conventionally, to synthesize such an array, the evolutionary algorithm is adopted to find out the optimum positions and on-times of the array elements simultaneously. Simultaneous consideration of the element positions and on-times as the optimization parameter vector increases the number of unknown parameters to be handled by the algorithm. The performance of the optimization algorithm is not well approved for handling large number of optimization parameters. For large arrays, the number of unknown parameters becomes so high that the optimization algorithms may not provide the desired power pattern by finding large unknown parameters. In this article, a method to synthesize UESTMAs with a comparatively smaller number of optimizing parameters has been investigated. In this regard, the array is synthesized in two steps by separately considering the element position and on-time of the array as the optimization parameters. It is worth to be noted that for the array with fixed element position, synthesis of a desired pattern by controlling element excitation is a linear array synthesis method. On the other hand, synthe-

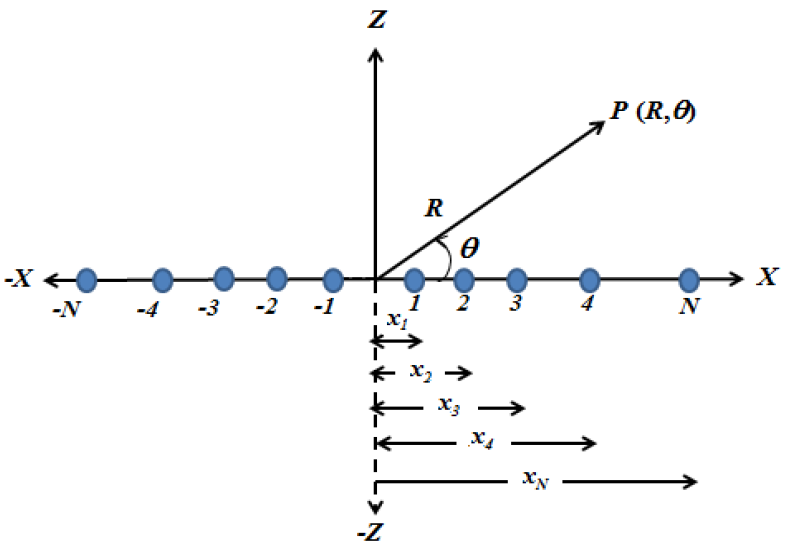

Fig. 1. A typical structure of a symmetric $2 N$-element unequally spaced linear array along $\mathrm{x}$-axis.

sis of antenna array with optimum element position is the non-linear array synthesis method [21]. Consequently, different cases of the non-linear and linear synthesizing methods such as position-only (PO), on-time only (OTO), on-time then position (OTTP), position then on-time (PTOT), and simultaneous position on-time (SPOT) have been studied. By employing three optimization algorithms namely, DE, TLBO and QPSO, it is shown that the performance of the two step PTOT synthesis method handling half of the optimization variables in each step is better or comparable to that of the conventional SPOT method holding all optimization variables together.

The rest of the paper is organized as follows. The theoretical analysis and problem formulation have been discussed in Sec. 2. Numerical result for the different synthesis method is presented in Sec. 3. Eventually, some conclusion about the superiority of the proposed method has been drawn in Sec. 4 .

\section{Theoretical Analysis and Problem Formulation}

\subsection{Theory}

A typical structure of a linear array consisting of $2 N$ isotropic elements, placed along the $\mathrm{x}$-axis is shown in Fig. 1. If the elements are located at $x_{1}, x_{2}, \ldots, x_{N}$, the corresponding array factor expression of such unequally spaced conventional antenna array (CAA) is written as,

$$
A F^{\mathrm{C}}(\theta)=\sum_{\substack{n=-N \\ n \neq 0}}^{N} A_{n} \mathrm{e}^{\mathrm{j} \alpha_{n}} \cdot \mathrm{e}^{\mathrm{j} \beta x_{n} \cos \theta} .
$$

Here, $A_{n}$ and $\alpha_{n}$ are the static excitation amplitude and phase of the $n^{\text {th }}$ element; $x_{n}$ is the position of the $n^{\text {th }}$ element from the reference position say origin of the coordinate system; $\beta=2 \pi / \lambda, \lambda$ is the wavelength; $\theta$ is the observation angle measured from the array axis. If each element in the array is connected with high-speed RF switches and the elements are periodically commutating with pre-specified 
on-time duration, the array factor expression in (1) is modified as [5],

$$
A F^{\mathrm{T}}(\theta, t)=\sum_{\substack{n=-N \\ n \neq 0}}^{N} A_{n} \mathrm{e}^{\mathrm{j} \alpha_{n}} U_{n}(t) \cdot \mathrm{e}^{\mathrm{j} \beta x_{n} \cos \theta} .
$$

In (2), $U_{n}(t)$ is the periodical time switching function with time modulation period, $T$ and over a complete period of time, it is expressed as,

$$
U_{n}(t)=\left\{\begin{array}{cc}
1, & 0 \leq t \leq \tau_{n} \leq T ; \quad \forall n \in[1, N] \\
0, & \text { otherwise }
\end{array}\right.
$$

where $\tau_{n}$ is the on-time duration of the $n^{\text {th }}$ element. As $U_{n}(t)$ is a periodical function of time, it can be decomposed in Fourier series to obtain the array factor at different harmonic components as,

$$
A F_{k}^{\mathrm{T}}(\theta, t)=\mathrm{e}^{\mathrm{j}\left\{2 \pi\left(f_{0}+k f_{\mathrm{m}}\right) t\right\}} \cdot \sum_{n=-N}^{N}\left(A_{n} \mathrm{e}^{\mathrm{j} \alpha_{n}}\right) \cdot C_{k n} \cdot \mathrm{e}^{\mathrm{j} \beta x_{n} \cos \theta}
$$

where $A F_{k}{ }^{\mathrm{T}}$ is the array factor at the $k^{\text {th }}$ harmonic, $f_{0}$ is the carrier signal frequency, $f_{\mathrm{m}}=1 / T$ is the modulation frequency and $C_{k n}$ is the Fourier coefficient of the $n^{\text {th }}$ element at the $k^{\text {th }}$ harmonic and is obtained as [10], [11],

$$
C_{k n}=\frac{A_{n} \tau_{n}}{T} \cdot \frac{\sin \left(\pi k f_{\mathrm{m}} \tau_{n}\right)}{\left(\pi k f_{\mathrm{m}} \tau_{n}\right)} \cdot \mathrm{e}^{-\mathrm{j} \pi k f_{\mathrm{m}} \tau_{n}}
$$

Considering uniform static excitation amplitude and phase with $A_{n}=1$, and $\alpha_{n}=0 ; \forall n \in[-N, N]$, the array factor expression at center frequency $f_{0}$ is obtained by combining (4) and (5) as,

$$
A F_{0}^{\mathrm{T}}(\theta, t)=\mathrm{e}^{\mathrm{j} 2 \pi f_{0} t} \cdot \sum_{n=-N}^{N} \frac{\tau_{n}}{T} \cdot \mathrm{e}^{\mathrm{j} \beta x_{n} \cos \theta} .
$$

From (6), it can be inferred that, by proper selection of the element positions $\left(x_{n}\right)$ and on-time durations $\left(\tau_{n}\right)$, the optimized power pattern with reduced SLL and SBL can be achieved.

\subsection{Problem Formulation}

In PO non-linear synthesis, the optimization aim is to suppress the SLL by finding the optimum element position whereas in OTO linear synthesis, only on-time of the antenna elements is optimized to realize power pattern with reduced SLL and SBL. In OTTP, first on-time is considered as the optimization parameter and then by setting the optimum on-time sequence as the on-time of the respective antenna elements, the element positions are optimized. In PTOT synthesis, first the element position is optimized and then by setting the element positions to this optimum value, on-time is taken as the optimization parameter vector. Thus, OTTP is linear to non-linear synthesis while PTOT is non-linear to linear synthesis. Finally, in SPOT synthesis, both the element position and on-time, i.e., non-linear, and linear parameters are simultaneously optimized to obtain the desired power pattern. Let us symbolize ' $b$ ' to denote the array synthesis method and ' $a$ ' as the desired design parameters of the antenna arrays. Thus, in PO synthesis, the array configuration becomes a conventional antenna arrays and the corresponding array factor as defined in (1) is used to synthesize the pattern by the minimizing the cost functions defined as,

$$
\psi=\sum_{a=1}^{2} W_{a}^{\mathrm{b}} \delta_{a}^{\mathrm{b}} \mathrm{H}_{a}\left(\delta_{a}^{\mathrm{b}}\right) .
$$

In (7), $\delta_{a}^{\mathrm{b}}$ with $a=1,2$ represents the difference between desired and obtained values of the design parameters of the respective beam as considered in the ' $\mathrm{b}=\mathrm{PO}$ ' synthesis method and is given as, $\delta_{1}^{\mathrm{b}}=\left|S L L_{\mathrm{d}}^{\mathrm{b}}-S L L_{\mathrm{o}}^{\mathrm{b}}\right|, \delta_{2}^{\mathrm{b}}=\left|F N B W_{\mathrm{d}}^{\mathrm{b}}-F N B W_{\mathrm{o}}^{\mathrm{b}}\right|$.

The same cost function is introduced for the OTO, OTTP, PTOT and SPOT synthesis methods and is given as,

$$
\psi=\sum_{a=1}^{2} W_{a}^{\mathrm{b}} \delta_{a}^{\mathrm{b}} \mathrm{H}_{a}\left(\delta_{a}^{\mathrm{b}}\right)+W^{\mathrm{b}} S B L_{\max }^{\mathrm{b}} .
$$

In (8), ' $b$ ' represents anyone of the other synthesis methods under consideration as OTO, OTTP, PTOT and SPOT. $\delta_{a}^{\text {b }}$ with $a=1,2$ represents the difference between the desired and obtained values of proper design specifications for the respective beam and is given as, $\delta_{1}^{\mathrm{b}}=\left|S L L_{\mathrm{d}}^{\mathrm{b}}-S L L_{\mathrm{o}}^{\mathrm{b}}\right|$ and $\delta_{2}^{\mathrm{b}}=\left|F N B W_{\mathrm{d}}^{\mathrm{b}}-F N B W_{\mathrm{o}}^{\mathrm{b}}\right|$; where $S L L_{\mathrm{o}}$ and $F N B W_{\mathrm{o}}$ represent the obtained values of SLL and beam-width between the first null of the main beam at center frequency $f_{0} . S L L_{\mathrm{d}}$, and $F N B W_{\mathrm{d}}$ are their respective desired values; $S B L_{\text {max }}^{\mathrm{b}}$ is the obtained maximum value of SBL in the different synthesis method; $W_{a}$ be the weighting factors of the related design. The weighting factor values used in the optimization process are 5 and 3, respectively. After several trials, the weighting factor values are predefined for the optimum results and these are remained constant for each case of synthesizing the array using different optimization algorithms [19], [20], [22-24]. $H_{a}$ is the Heaviside step function. This is used to compare the obtained values of intended radiation parameters with their respective desired values during the process of optimization such that, the function value is 1 (one) as long as the intended radiation parameter doesn't reach to its desired value while the function value becomes 0 (zero) whenever the obtained values of intended radiation parameters are better than or equal to their respective desired values.

\section{Results and Discussion}

In this section, various simulation results are performed to establish the superiority and effectiveness of the proposed approach. Let us consider a 32-element linear array with isotropic elements symmetrically placed around the array center. In [12], a similar array is synthesized by simultaneously considering element position and on-time as the optimization parameters. Due to symmetry, total number 
of optimization parameters becomes 32 with 16 for the element position and 16 for the on-time duration. Here, with the aim of synthesizing the array with smaller optimization parameter, a two steps synthesis method is investigated. In this regard, three algorithms DE, TLBO and QPSO are applied to synthesize the array under different cases as detailed in the following sections. The tuning parameters of the algorithms are set for their optimum performance as detailed in [20-24]. Here, the tuning parameters of $\mathrm{DE}$ are set as mutation intensity $F=0.5$, crossover probability $\eta c=0.85$. As considered in [12], the search range of the optimization parameters are defined as element position $(0.55,1)$, and on-time $(0.01,1)$. To synthesize a center frequency pattern, the desired value of SLL and FNBW in the cost functions are set to $S L L_{\mathrm{d}}=-30 \mathrm{~dB}$ and $F N B W_{\mathrm{d}}=10^{\circ}$ respectively while SBL to be suppressed as much low as possible. For a fair comparison of the algorithms and the synthesis methods under consideration, for each case, the population size and maximum number of iterations are kept same for all three algorithms as $N P_{\mathrm{DE}}=$ $N P_{\text {TLBO }}=N P_{\text {QPSO }}=3 \mathrm{D}$ and $g_{\max }=1000$. Here, 'D' stands for the dimension of the problem i.e., for the array synthesis problem under consideration, number of unknown parameters to be determined and $g_{\max }$ is the maximum number of generation index of the used evolutionary algorithm for evolving the population of the possible solution.

\subsection{Case 1: Position-Only (PO)}

As mentioned in Sec. 2.2, for PO non-linear type synthesis, only element position is considered as the optimization parameter and the corresponding cost function as defined in (7) is used to synthesize the array. The DE, TLBO and QPSO optimized power pattern at center frequency is shown in Fig. 2 and the corresponding elementwise position is depicted in Tab. 1. The returned values of SLL and FNBW are shown in Tab. 2. It can be seen that the obtained values of SLLs of PO synthesized patterns under different algorithms are much higher than the desired value. As compared to TLBO and QPSO, SLL of the DE optimized pattern is suppressed slightly more, about $1 \mathrm{~dB}$. However, this leads to increase the beam-width by 0.4 degree with respect to the others. In this context, this is

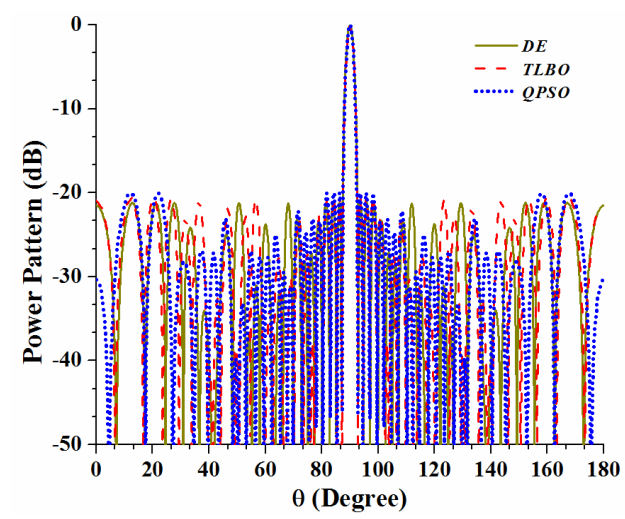

Fig. 2. Radiation pattern for PO synthesis of 32 element linear array at center frequency, $f_{0}$.

\begin{tabular}{|c|c|c|c|}
\hline \multirow{2}{*}{$\begin{array}{c}\text { Element No. } \\
(\boldsymbol{n})\end{array}$} & \multicolumn{3}{|c|}{ Element position $\left(\boldsymbol{x}_{\boldsymbol{n}}\right)$ in terms of $\boldsymbol{\lambda}$} \\
\cline { 2 - 4 } & DE & TLBO & QPSO \\
\hline \pm 1 & \pm 0.275 & \pm 0.324 & \pm 0.275 \\
\hline \pm 2 & \pm 0.827 & \pm 0.874 & \pm 0.826 \\
\hline \pm 3 & \pm 1.377 & \pm 1.491 & \pm 1.399 \\
\hline \pm 4 & \pm 1.928 & \pm 2.172 & \pm 2.005 \\
\hline \pm 5 & \pm 2.532 & \pm 2.773 & \pm 2.660 \\
\hline \pm 6 & \pm 3.167 & \pm 3.527 & \pm 3.386 \\
\hline \pm 7 & \pm 3.786 & \pm 4.354 & \pm 4.021 \\
\hline \pm 8 & \pm 4.575 & \pm 5.225 & \pm 4.624 \\
\hline \pm 9 & \pm 5.398 & \pm 6.134 & \pm 5.364 \\
\hline \pm 10 & \pm 6.277 & \pm 6.987 & \pm 6.197 \\
\hline \pm 11 & \pm 7.150 & \pm 7.904 & \pm 7.077 \\
\hline \pm 12 & \pm 8.023 & \pm 8.768 & \pm 7.946 \\
\hline \pm 13 & \pm 8.930 & \pm 9.325 & \pm 8.827 \\
\hline \pm 14 & \pm 9.743 & \pm 9.876 & \pm 9.727 \\
\hline \pm 15 & \pm 10.31 & \pm 10.706 & \pm 10.595 \\
\hline \pm 16 & \pm 11.09 & \pm 11.686 & \pm 11.456 \\
\hline
\end{tabular}

Tab. 1. Element-wise position obtained using different algorithms.

\begin{tabular}{|c|c|c|c|c|c|}
\hline \multirow{2}{*}{$\begin{array}{c}\text { Synthesis } \\
\text { Method }\end{array}$} & Parameters & \multirow{2}{*}{$\begin{array}{c}\text { Desired } \\
\text { Values }\end{array}$} & \multicolumn{3}{|c|}{ Obtained Values } \\
\cline { 4 - 6 } & & DE & TLBO & QPSO \\
\hline \multirow{4}{*}{ PO } & SLL(dB) & -30 & -21.15 & -20.47 & -20.02 \\
\cline { 2 - 6 } & SBL (dB) & - & - & - & - \\
\cline { 2 - 6 } & FNBW (degree) & 10 & 6 & 5.6 & 5.6 \\
\cline { 2 - 6 } & Computation time (s) & & 166.5 & 160.6 & 171.6 \\
\hline \multirow{3}{*}{ OTO } & SLL(dB) & -30 & -20 & -19.51 & -19.44 \\
\cline { 2 - 6 } & SBL (dB) & - & -27.91 & -27.41 & -22.92 \\
\cline { 2 - 6 } & FNBW (degree) & 10 & 9.6 & 10 & 10.2 \\
\cline { 2 - 6 } & Computation time (s) & & 705.8 & 704.08 & 709.2 \\
\hline
\end{tabular}

Tab. 2. Comparative results of PO and OTO synthesis using different algorithms.

to be mentioned that a similar array is synthesized by optimizing element position in [3], [4] and almost the same result is obtained. This indicates that the obtained positions of the array elements are corresponding to one possible optimum solution of the synthesized pattern.

\subsection{Case 2: On-time Only (OTO)}

In linear OTO synthesis, only on-time duration of the time-modulated array elements are optimized by considering the array as one equally spaced array. Without loss of any generality, the inter-element spacing of the array is taken as $0.5 \lambda$. The corresponding to the individual algorithm, the normalized far-field pattern at center frequency and the $1^{\text {st }}$ sideband frequency pattern are shown in Figs. 3(a) and (b), respectively. The optimized element wise ontime sequence is presented in Tab. 3. Along with the PO synthesis results, the obtained values of SLL, SBL and FNBW are also given in Tab. 2. From Tab. 2 it is evident that the obtained values of SLL for both PO and OTO synthesized patterns with different algorithms are closed to each other's. However, in PO synthesis while element position is optimized, here in OTO synthesis only the ontime sequence is optimized for the equally spaced array. It can be observed that, like PO synthesis approach, the obtained value of SLLs in OTO synthesis is much higher than the desired values. When the obtained patterns using DE, 


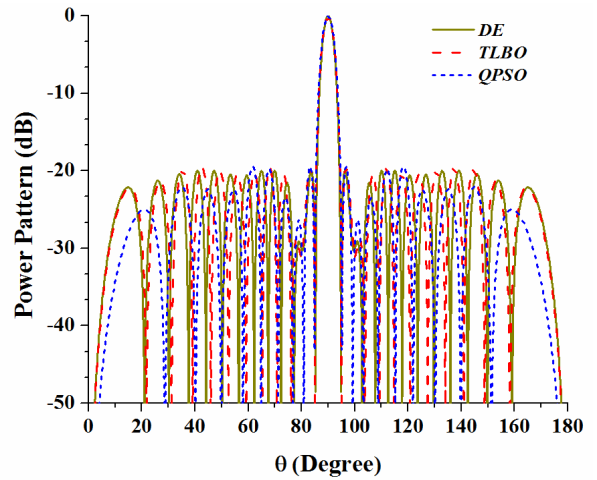

(a)

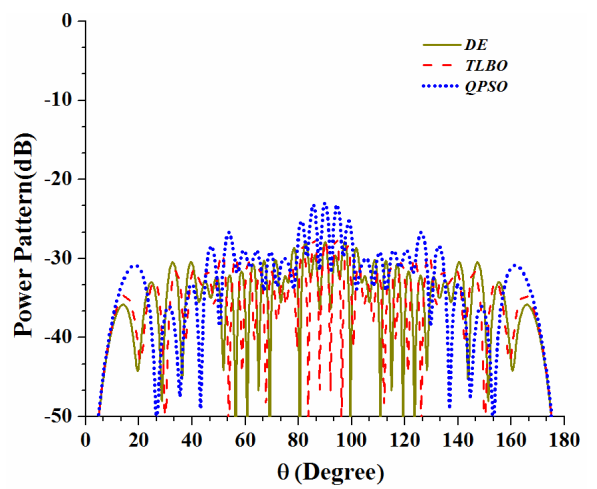

(b)

Fig. 3. (a) Radiation pattern for OTO synthesis of 32 element linear array at center frequency, $f_{0}$. (b) Radiation patterns at the $1^{\text {st }}$ sideband frequency, $f_{0}+f_{\mathrm{p}}$, for 32 element linear array.

\begin{tabular}{|c|c|c|c|}
\hline \multirow{2}{*}{$\begin{array}{c}\text { Element No } \\
(\boldsymbol{n})\end{array}$} & \multicolumn{3}{|c|}{ Normalized on-time durations $\left(\boldsymbol{\tau}_{\boldsymbol{n}}\right)$} \\
\cline { 2 - 4 } & $\mathbf{D E}$ & TLBO & QPSO \\
\hline \pm 1 & 1 & 1 & 1 \\
\hline \pm 2 & 1 & 1 & 0.999 \\
\hline \pm 3 & 1 & 1 & 0.999 \\
\hline \pm 4 & 1 & 0.944 & 0.995 \\
\hline \pm 5 & 1 & 0.936 & 0.985 \\
\hline \pm 6 & 0.998 & 0.824 & 0.980 \\
\hline \pm 7 & 1 & 0.809 & 0.668 \\
\hline \pm 8 & 0.949 & 0.751 & 0.947 \\
\hline \pm 9 & 0.901 & 0.667 & 0.216 \\
\hline \pm 10 & 0.926 & 0.454 & 0.861 \\
\hline \pm 11 & 0.823 & 0.606 & 0.872 \\
\hline \pm 12 & 0.020 & 0.321 & 0.926 \\
\hline \pm 13 & 0.013 & 0.288 & 0.256 \\
\hline \pm 14 & 0.988 & 0.314 & 0.068 \\
\hline \pm 15 & 0.095 & 0.282 & 0.275 \\
\hline \pm 16 & 0.403 & 0.247 & 0.070 \\
\hline
\end{tabular}

Tab. 3. Element-wise on-time sequence obtained using different algorithms.

TLBO and QPSO are compared, the DE optimized pattern is improved by about $0.5 \mathrm{~dB}$ and 0.4 degree less values of SLL and beamwidth, respectively. However, for all the algorithms, the average computation time to synthesize the array is almost same. In [8], by considering a 30-element time-modulated linear array (TMLA) with $d=0.7 \lambda$, almost an equivalent result is obtained. Therefore, this implies that the obtained solution as the on-time duration of the respective array element belongs to one possible optimum solution of this array synthesis problem.

\subsection{Case 3: On-time then Position (OTTP)}

With the objective to synthesize the unequally spaced time-modulated linear array (UESTMLA) with a smaller number of optimization variables, the array is synthesized in two steps. In OTTP synthesis method, first the on-time is considered as optimization parameter then with the optimized on-time sequence, the element position is optimized. Since, the first step of synthesizing the array is same as in Case 2, therefore, with the optimized on-time values as presented in Tab. 3, DE, TLBO and QPSO algorithms are employed to determine the element positions. The optimized elementwise on-time sequence of the algorithms is presented in Tab. 4. The center frequency pattern as obtained with different algorithms is shown in Fig. 4(a) and the corresponding relative $1^{\text {st }}$ sideband frequency pattern with respect to the maximum of the center frequency pattern is shown in Fig. 4(b). The obtained values of SLL, SBL and FNBW using different algorithms are depicted in Tab. 5. It can be observed that compared to Case 1 and Case 2, the SLL of the synthesized pattern is not improved significantly. However, in [12] taking both the variables together as the optimization parameters, SLL and SBL of the synthesized pattern with one optimization step are reduced to $-30 \mathrm{~dB}$ and $-22.7 \mathrm{~dB}$, respectively. Thus, it reveals that, in this two-step i.e., linear to non-linear synthesis method, consideration of ontime in the first step and then the element position in the second step is not effective to improve the radiation pattern satisfactorily.

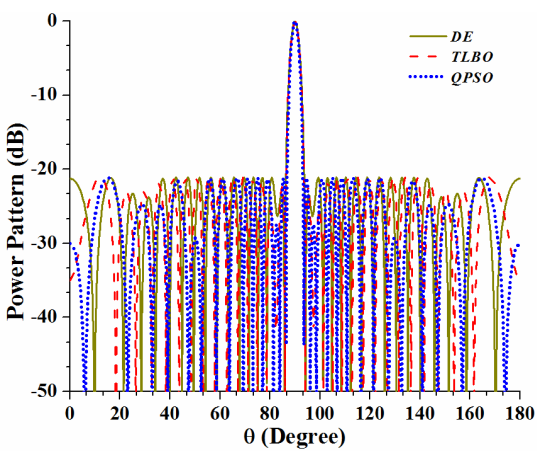

(a)

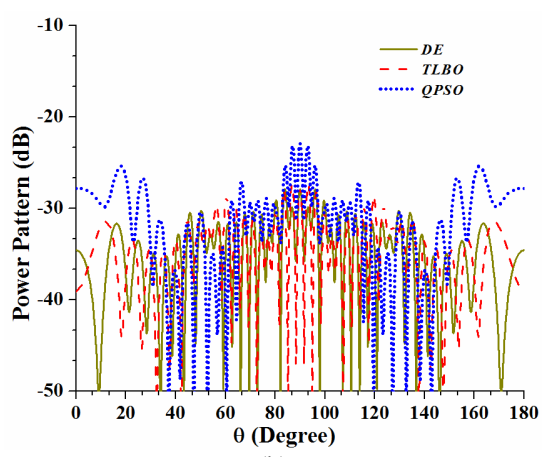

(b)

Fig. 4. (a) Radiation pattern for OTTP synthesis of 32 element linear array at center frequency, $f_{0}$. (b) Radiation patterns at the $1^{\text {st }}$ sideband, $f_{0}+f_{\mathrm{p}}$, corresponding to the center frequency pattern of Fig. 4(a). 


\subsection{Case 4: Position then On-time (PTOT)}

In PTOT synthesis, in the first step, the element position is considered as optimization parameter and then, by setting the element positions same as obtained in the first step, on-time durations are optimized in the second step. It can be seen that the first step of the synthesis method is same as considered in Case 1. Therefore, taking the element position of Tab. 1, the array is synthesized by optimizing the on-time duration of elements. The optimized element-wise positions and on-time values of the respective algorithms are depicted in Tab. 4. Figures 5(a) and (b) show the obtained radiation pattern at center frequency, $f_{0}$ and the $1^{\text {st }}$ sideband, respectively. The obtained values of SLL, SBL and FNBW using different algorithms are shown in Tab. 5. For all the algorithms, the desired value of SLL is achieved as $-30 \mathrm{~dB}$ while SBLs with DE, TLBO and QPSO are obtained as $-24.12 \mathrm{~dB},-22.63 \mathrm{~dB}$ and $-19.93 \mathrm{~dB}$, respectively. Hence, an equivalent optimized pattern as realized in [12] is obtained. This indicates that, along with low value of SLL, significant suppression of $\mathrm{SBL}$ is observed under this non-linear to linear synthesis method.

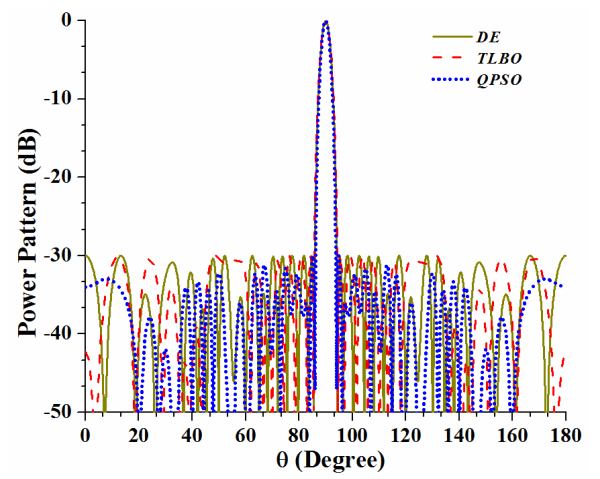

(a)

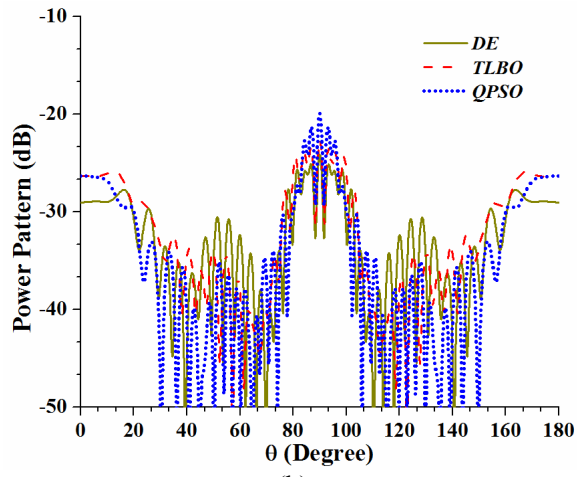

(b)

Fig. 5. (a) Radiation pattern for PTOT synthesis of 32 element linear array at center frequency, $f_{0}$. (b) Radiation patterns at the $1^{\text {st }}$ sideband frequency, $f_{0}+f_{\mathrm{p}}$, for 32 element linear array.

\begin{tabular}{|c|c|c|c|c|c|}
\hline \multirow{2}{*}{$\begin{array}{l}\text { Synthesis } \\
\text { Method }\end{array}$} & \multirow{2}{*}{ Parameters } & \multirow{2}{*}{$\begin{array}{l}\text { Desired } \\
\text { Values }\end{array}$} & \multicolumn{3}{|c|}{ Obtained Values } \\
\hline & & & $\overline{D E}$ & TLBO & QPSO \\
\hline \multirow{4}{*}{ OTTP } & SLL(dB) & -30 & -21.29 & -21.08 & -21.17 \\
\hline & SBL (dB) & - & -27.9 & -27.37 & -22.92 \\
\hline & FNBW (deg) & 10 & 8.6 & 8 & 7.2 \\
\hline & $\begin{array}{c}\text { Computation time } \\
\text { (s) }\end{array}$ & & 727.2 & 714.3 & 723.4 \\
\hline \multirow{4}{*}{ PTOT } & SLL(dB) & -30 & -30 & -30 & -30 \\
\hline & SBL (dB) & - & -24.12 & -22.63 & -19.93 \\
\hline & FNBW (deg) & 10 & 8.8 & 9.2 & 8.2 \\
\hline & $\begin{array}{c}\text { Computation time } \\
\text { (s) }\end{array}$ & & 743.8 & 690.3 & 755.9 \\
\hline \multirow{4}{*}{ SPOT } & SLL(dB) & -30 & -30 & -30 & -30 \\
\hline & SBL (dB) & - & -23.97 & -23.91 & -15.42 \\
\hline & FNBW (deg) & 10 & 8.6 & 9.8 & 7.8 \\
\hline & $\begin{array}{c}\text { Computation time } \\
\text { (s) }\end{array}$ & & 1394.9 & 1357.6 & 1580.7 \\
\hline
\end{tabular}

Tab. 5. Comparative results of SPOT, OTTP and PTOT synthesis using different algorithms.

\begin{tabular}{|c|c|c|c|c|c|c|c|c|c|c|c|c|}
\hline \multirow{3}{*}{$\begin{array}{c}\text { Element } \\
\text { No. } \\
(\boldsymbol{n})\end{array}$} & \multirow{2}{*}{\multicolumn{3}{|c|}{$\begin{array}{c}\text { OTTP } \\
\text { With the same on-time }\left(\tau_{n}\right) \text { as } \\
\text { given in Tab. 3, the optimized } \\
\text { element position }\left(x_{n}\right) \text { under } \\
\text { different algorithm }\end{array}$}} & \multirow{2}{*}{\multicolumn{3}{|c|}{\begin{tabular}{|c|} 
PTOT \\
With the same element \\
position $\left(x_{n}\right)$ as given in Tab. 1, \\
the optimized on-time $\left(\tau_{n}\right)$ \\
under different algorithm \\
\end{tabular}}} & \multicolumn{6}{|c|}{ SPOT } \\
\hline & & & & & & & \multicolumn{2}{|c|}{ DE } & \multicolumn{2}{|c|}{ TLBO } & \multicolumn{2}{|c|}{ QPSO } \\
\hline & DE & TLBO & QPSO & DE & TLBO & QPSO & $\begin{array}{l}\text { Element } \\
\text { position } \\
\left(x_{n}\right)\end{array}$ & $\begin{array}{c}\text { On-time } \\
\left(\tau_{n}\right)\end{array}$ & $\begin{array}{c}\text { Element } \\
\text { position } \\
\left(x_{n}\right)\end{array}$ & $\begin{array}{l}\text { On-time } \\
\quad\left(\tau_{n}\right)\end{array}$ & $\begin{array}{c}\text { Element } \\
\text { position } \\
\left(x_{n}\right)\end{array}$ & $\begin{array}{c}\text { On-time } \\
\left(\tau_{n}\right)\end{array}$ \\
\hline \pm 1 & \pm 0.305 & \pm 0.324 & \pm 0.376 & 0.999 & 0.997 & 0.999 & \pm 0.275 & 0.998 & \pm 0.275 & 0.999 & \pm 0.317 & 0.998 \\
\hline \pm 2 & \pm 0.904 & \pm 0.973 & \pm 1.091 & 1 & 0.999 & 0.999 & \pm 0.826 & 0.997 & \pm 0.846 & 0.999 & \pm 1.035 & 0.999 \\
\hline \pm 3 & \pm 1.497 & \pm 1.657 & \pm 1.851 & 0.999 & 0.999 & 0.999 & \pm 1.381 & 0.994 & \pm 1.396 & 0.996 & \pm 1.818 & 0.999 \\
\hline \pm 4 & \pm 2.064 & \pm 2.375 & \pm 2.644 & 0.995 & 0.983 & 0.999 & \pm 1.937 & 0.989 & \pm 2.010 & 0.999 & \pm 2.603 & 0.992 \\
\hline \pm 5 & \pm 2.626 & \pm 3.055 & \pm 3.376 & 1 & 0.999 & 0.999 & \pm 2.527 & 0.998 & \pm 2.638 & 0.971 & \pm 3.350 & 0.925 \\
\hline \pm 6 & \pm 3.178 & \pm 3.730 & \pm 4.198 & 0.964 & 0.999 & 0.989 & \pm 3.183 & 0.986 & \pm 3.245 & 0.992 & \pm 4.032 & 0.902 \\
\hline \pm 7 & \pm 3.742 & \pm 4.441 & \pm 4.942 & 0.999 & 0.999 & 0.912 & \pm 3.815 & 0.988 & \pm 4.006 & 0.999 & \pm 4.768 & 0.883 \\
\hline \pm 8 & \pm 4.300 & \pm 5.154 & \pm 5.577 & 0.999 & 0.897 & 0.905 & \pm 4.580 & 0.991 & \pm 4.847 & 0.932 & \pm 5.556 & 0.837 \\
\hline \pm 9 & \pm 4.879 & \pm 5.860 & \pm 6.350 & 0.957 & 0.824 & 0.930 & \pm 5.420 & 0.970 & \pm 5.707 & 0.835 & \pm 6.377 & 0.724 \\
\hline \pm 10 & \pm 5.590 & \pm 6.530 & \pm 7.153 & 0.812 & 0.693 & 0.880 & \pm 6.276 & 0.879 & \pm 6.586 & 0.828 & \pm 7.212 & 0.520 \\
\hline \pm 11 & \pm 6.365 & \pm 7.206 & \pm 8.000 & 0.819 & 0.321 & 0.801 & \pm 7.163 & 0.783 & \pm 7.470 & 0.360 & \pm 7.979 & 0.406 \\
\hline \pm 12 & \pm 7.035 & \pm 7.900 & \pm 8.802 & 0.561 & 0.162 & 0.567 & \pm 8.030 & 0.632 & \pm 8.315 & 0.206 & \pm 8.747 & 0.395 \\
\hline \pm 13 & \pm 7.590 & \pm 8.477 & \pm 9.547 & 0.150 & 0.122 & 0.493 & \pm 8.961 & 0.197 & \pm 8.865 & 0.091 & \pm 9.535 & 0.263 \\
\hline \pm 14 & \pm 8.245 & \pm 9.155 & \pm 10.115 & 0.195 & 0.105 & 0.271 & \pm 9.837 & 0.224 & \pm 9.415 & 0.111 & \pm 10.396 & 0.251 \\
\hline \pm 15 & \pm 8.796 & \pm 9.970 & \pm 10.899 & 0.074 & 0.067 & 0.177 & \pm 10.65 & 0.125 & \pm 10.211 & 0.056 & \pm 11.173 & 0.137 \\
\hline \pm 16 & \pm 9.346 & \pm 10.71 & \pm 11.610 & 0.094 & 0.028 & 0.197 & \pm 11.20 & 0.080 & \pm 11.219 & 0.053 & \pm 12.009 & 0.220 \\
\hline
\end{tabular}

Tab. 4. Position and on-time distribution obtained in different cases using DE, TLBO and QPSO. 


\subsection{Case 5: Simultaneous Position and On- time (SPOT)}

To examine the performance of the algorithms in synthesizing the array with simultaneous position and ontime (SPOT) as the optimization parameters, the same algorithms are used to synthesize the array in a single step by optimizing both the parameters together. As both the array design variables are optimized simultaneously, the number of parameters to be optimized in this case becomes 32. With the optimized element position and on-time of the respective algorithms as presented in Tab. 4, the synthesized radiation patterns at $f_{0}$ are shown in Fig. 6(a), while the relative $1^{\text {st }}$ sideband frequency pattern are depicted in Fig. 6(b). The calculated SLL, SBL and FNBW of the patterns using DE, TLBO and QPSO are given in Tab. 5. It can be seen that obtained radiation patterns are closed to that achieved under PTOT. However, in the two steps of PTOT synthesis method, the computation time for synthesizing the array using DE, TLBO and QPSO can be calculated from Tab. 2 and Tab. 5 and are obtained as 910.3 $(743.8+166.5)$ seconds; $850.9(690.3+160.6)$ seconds and $927.7(755.9+171.6)$ seconds, respectively. The computation time of the respective algorithms with SPOT synthesis methods are 1394.9, 1357.6 and 1580.7 seconds, respectively. This result indicates that the computation time for the PTOT synthesis method is significantly less than that for the conventional SPOT synthesis method. While the performances of three algorithms are compared, both DE

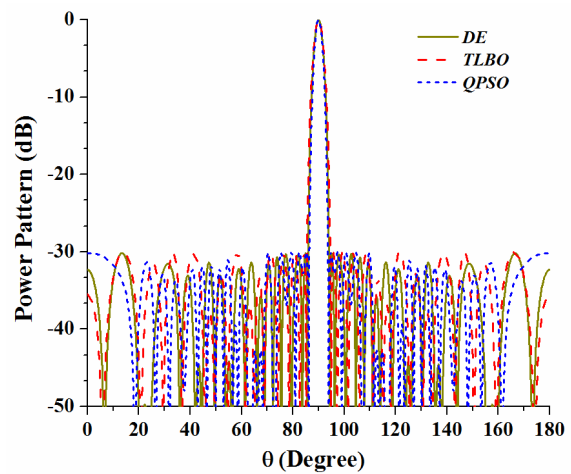

(a)

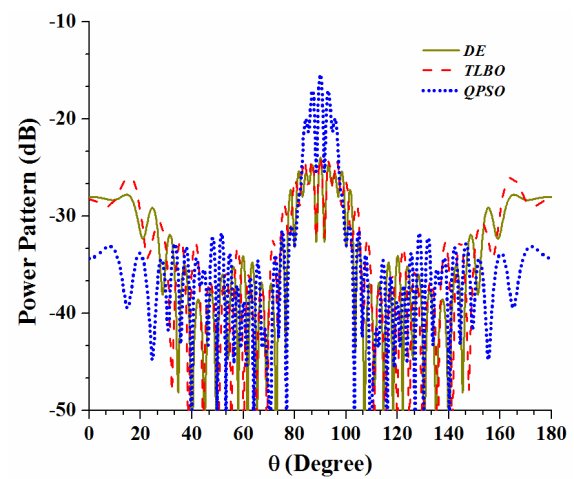

(b)

Fig. 6. (a) Radiation pattern for SPOT synthesis of 32 element linear array at center frequency, $f_{0}$. (b) Radiation patterns at the $1^{\text {st }}$ sideband frequency, $f_{0}+f_{\mathrm{p}}$, for 32 element linear array. and TLBO outperform over QPSO in all the cases of the synthesis methods. Though radiation parameters (SLL, FNBW, and SBL) of the synthesized patterns using DE are better as compared to that obtained by using TLBO, the computation time taken by TLBO is smaller than DE. Hence, one can use DE to synthesize radiation patterns, while TLBO can be utilized to achieve radiation pattern closed to that of DE with less computation time. This further validates the effectiveness the PTOT method to synthesize UESTMA with less computational burden as compared to SPOT.

\section{Conclusion}

A two-step synthesis method with fewer unknown parameters in each step is presented to synthesize UESTMAs. Though there are two possible two-step synthesis methods - linear to non-linear i.e., OTTP and non-linear to linear i.e., PTOT, however, out of these two, PTOT is effective to synthesize power pattern with improved radiation characteristics, specifically, narrow beam pattern with reduced SLL and SBL. For synthesizing the power patterns with desired radiation parameters, performance of PTOT is closed to that of the conventionally used SPOT. However, consideration of both position and on-time as the design parameters in SPOT leads to increase the problem dimension and hence the search space to find the solution. This leads to increase the computational time to obtain the optimum solution in SPOT synthesis method. When the performance of the three used algorithms are compared, DE and TLBO outperform over QPSO. While the radiation parameters of DE and TLBO synthesized patterns are closed to each other, the convergence rate of TLBO algorithm is better than the other two algorithms. One of the main advantages of TLBO algorithm is that it requires only the common controlling parameters such as number of generation and population size. As in the proposed PTOT synthesis method, the problem dimension becomes half, with respect to the traditional SPOT synthesis method, PTOT will be beneficial to synthesize large antenna arrays. Finally, this method can be extended to synthesize other geometries of antenna arrays.

\section{Acknowledgment}

This work is financially supported by the Science and Engineering Research Board (SERB), Govt. of India, and the work is under the project Ref. file number EEQ/2016/00836, dated January 17, 2017.

\section{References}

[1] ELliOTT, R. S. Antenna Theory and Design. Englewood Cliffs (N.J., USA): Prentice-Hall, 1981. ISBN: 9780470544174

[2] LIN, C., QING, A., FENG, Q. Synthesis of unequally spaced antenna arrays by using differential evolution. IEEE Transactions 
on Antennas and Propagation, 2010, vol. 58, no. 8, p. 2553-2561. DOI: $10.1109 /$ TAP.2010.2048864

[3] KURUP, D. G., HIMDI, M., RYDBERG, A. Synthesis of uniform amplitude unequally spaced antenna arrays using the differential evolution algorithm. IEEE Transactions on Antennas and Propagation, 2003, vol. 51, no. 9, p. 2210-2217. DOI: 10.1109/TAP.2003.816361

[4] AHMAD, A., BEHERA, A. K., MANDAL, S. K., et al. Artificial bee colony algorithm to reduce the side lobe level of uniformly excited linear antenna arrays through optimized element spacing. In IEEE Conference on Information \& Communication Technologies. Thuckalay (India), 2013, p. 1029-1032. DOI 10.1109/CICT.2013.6558249

[5] YOU, P., LIU, Y., CHEN, S., et al. Synthesis of unequally spaced linear antenna arrays with minimum element spacing constraint by alternating convex optimization. IEEE Antennas and Wireless Propagation Letters, 2017, vol. 16, p. 3126-3130. DOI: 10.1109/LAWP.2017.2764069

[6] SHANKS, H. E., BICKMORE, R. W. Four-dimensional electromagnetic radiators. Canadian Journal of Physics, 1959, vol. 37, no. 3, p. 263-275. DOI: 10.1139/p59-031

[7] HE, C., WANG, L., CHEN, J., et al. Time-modulated arrays: A four-dimensional antenna array controlled by switches. Journal of Communications and Information Networks, 2018, vol. 3, no. 1, p. 1-14. DOI: $10.1007 / \mathrm{s} 41650-018-0004-7$

[8] FONDEVILA, J., BREGAINS, J. C., ARES, F., et al. Optimizing uniformly excited linear arrays through time modulation. IEEE Antennas and Wireless Propagation Letters, 2004, vol. 3, p. 298-301. DOI: 10.1109/LAWP.2004.838833

[9] KUMMER, W., VILLENEUVE, A., FONG, T., et al. Ultra-low sidelobes from time-modulated arrays. IEEE Transactions on Antennas and Propagation, 1963, vol. 11, no. 6, p. 633-639. DOI: 10.1109/TAP.1963.1138102

[10] FONDEVILA, J., BREGAINS, J.C., ARES, F., et al. Application of time modulation in the synthesis of sum and difference patterns by using linear arrays. Microwave and Optical Technology Letters, 2006, vol. 48, no. 5, p. 829-832. DOI: 10.1002/mop.21489

[11] YANG, S., GAN, Y. B., TAN, P. K. A new technique for powerpattern synthesis in time-modulated linear arrays. IEEE Antennas and Wireless Propagation Letters, 2003, vol. 2, p. 285-287. DOI: 10.1109/LAWP.2003.821556

[12] LI, G., YANG, S., HUANG, M., et al. Sidelobe suppression in time modulated linear arrays with unequal element spacing. Journal of Electromagnetic Waves and Applications, 2012, vol. 24 , no. 5-6, p. 775-783. DOI: $10.1163 / 156939310791036368$

[13] MANDAL, S. K., MAHANTI, G. K., GHATAK, R. Differential evolution algorithm for optimizing the conflicting parameters in time-modulated linear array antennas. Progress In Electromagnetics Research, PIER B, 2013, vol. 51, p. 101-118. DOI: 10.2528/PIERB13022710

[14] PATRA, S., MANDAL, S. K., MAHANTI, G. K., et al. Synthesis of flat-top power pattern in time-modulated unequally spaced linear arrays using DE. In IEEE 2nd International Conference on Recent Trends in Information Systems (ReTIS). Kolkata (India), 2015, p. 104-108. DOI: 10.1109/ReTIS.2015.7232861

[15] YANG, S., GAN, Y. B., QING, A., et al. Design of a uniform amplitude time modulated linear array with optimized time sequences. IEEE Transactions on Antennas and Propagation, 2005, vol. 53, no. 7, p. 2337-2339. DOI: 10.1109/TAP.2005.850765

[16] POLI, L., ROCCA, P., OLIVERI, G., et al. Harmonic beamforming in time-modulated linear arrays. IEEE Transactions on Antennas and Propagation, 2011, vol. 59, no. 7, p. 2538-2545. DOI: 10.1109/TAP.2011.2152323
[17] ZHANG, S. R., ZHANG, Y. X., CUI, C. Y. Efficient multiobjective optimization of time-modulated array using a hybrid particle swarm algorithm with convex programming. IEEE Antennas and Wireless Propagation Letters, 2020, vol. 19, no. 11, p. 1842-1846. DOI: 10.1109/LAWP.2020.3014366

[18] MANDAL, S. K., MAHANTI, G. K., GHATAK, R. Synthesis of simultaneous multiple-harmonic-patterns in time-modulated linear antenna arrays. Progress In Electromagnetics Research M, 2014 vol. 34, p. 135-142. DOI: 10.2528/PIERM13111802

[19] RAO, R. V., SAVSANI, V. J., VAKHARIA, D. P. Teachinglearning-based optimization: A novel method for constrained mechanical design optimization problems. Computer-Aided Design, 2011, vol. 43, no. 3, p. 303-315. DOI: 10.1016/j.cad.2010.12.015

[20] RAO, R. V., SAVSANI, V. J., VAKHARIA, D. P. Teachinglearning-based optimization: An optimization method for continuous non-linear large scale problems. Information Sciences, 2012, vol. 183, no. 1, p. 1-15. DOI: 10.1016/j.ins.2011.08.006

[21] KUMAR, B. P., BRANNER, G. R. Generalized analytical technique for the synthesis of unequally spaced arrays with linear, planar, cylindrical or spherical geometry. IEEE Transactions on Antennas and Propagation, 2005, vol. 53, no. 2, p. 621-634. DOI: 10.1109/TAP.2004.841324

[22] STORN, R., PRICE, K. Differential evolution - A simple and efficient heuristic for global optimization over continuous spaces. Journal of Global Optimization, 1997, vol. 11 no. 4, p. 341-359. DOI: $10.1023 / \mathrm{A}: 1008202821328$

[23] SUN, J., XU, W., FENG, B. A global search strategy of quantumbehaved particle swarm optimization. In IEEE Conference on Cybernetics and Intelligent Systems, Singapore, 2004, p. 111-116. DOI: $10.1109 /$ ICCIS.2004.1460396

[24] SUN, J., FANG, W., PALADE, V., et al. Quantum-behaved particle swarm optimization with Gaussian distributed local attractor point. Applied Mathematics and Computation, 2011, vol. 218, no. 7, p. 3763-3775. DOI: 10.1016/j.amc.2011.09.021

\section{About the Authors ...}

Somnath PATRA received B-Tech \& M-Tech degree from WBUT in 2008 \& 2011, respectively. He is currently pursuing his Ph.D. from NIT Durgapur, India. He has more than 8 years of teaching experience.

Sujit Kumar MANDAL received B.Sc. degree in Physics (H) from the University of Calcutta in 2001. He completed B.Tech. and M.Tech. in Radio Physics \& Electronics from C. U. in the year 2004 and 2006, respectively. He received Ph.D. degree in the year 2014 from NIT, Durgapur. Presently, he is working as Assistant Professor in the Dept. of ECE, NIT Durgapur, India. He has published more than 50 research papers in national and internal peer reviewed journals and conferences.

Gautam Kumar MAHANTI received B.E. in ECE from NIT Durgapur in 1988, M.E. in Electronics System and Communication from NIT, Rourkela in 1994, and Ph.D. from IIT, Kharagpur, India. He has more than 20 years of teaching and research experience. Presently, he is working as Professor in the Dept. of ECE, NIT, Durgapur, India.

Narendra Nath PATHAK received his Ph.D. degree from NIT Durgapur and is currently working as Professor and HOD in Dr. B. C. Roy Eng. College, Durgapur, India. 